\title{
IDENTIFICATION OF ANALYTICAL DEPENDENCIES OF THE OPERATIONAL CHARACTERISTICS OF THE WORKPIECE CLAMPING MECHANISMS WITH THE ROTARY MOVEMENT OF THE INPUT LINK
}

\author{
Borys PRYDALNYI*, Heorhiy SULYM** \\ *Faculty of Technology, Department of Applied mechanics, Lutsk National Technical University, Lvivska Str 75, 43018 Lutsk, Ukraine \\ ${ }^{* *}$ Faculty of Mechanical Engineering, Bialystok University of Technology, ul. Wiejska 45C, 15-351 Bialystok, Poland \\ prydalnyy30@gmail.com, h.sulym@pb.edu.pl
}

received 7 October 2019, revised 5 May 2021, accepted 10 May 2021

\begin{abstract}
The research is devoted to the problem of determining the efficiency of the workpiece fixing mechanism operation. Improving characteristics of workpiece fixing is one of the required conditions to increase the cutting modes, which may help to enhance the machining productivity. The study investigates the main characteristics and general features of a new structure of clamping mechanisms with electromechanical actuators for fixation of rotation bodies. The main advantages of using electromechanical clamping actuators with self-braking gear are presented. Two simplified dynamical models for the description of different stages of the clamping process are developed. The calculation scheme was formulated to find out how the mass-geometric parameters of mechanism elements should influence the main characteristics of the clamping mechanisms of this type.
\end{abstract}

Keywords: the mechanism for clamping of rotation bodies, clamping actuator, self-braking screw gear, kinematic characteristics, workpiece clamping efficiency

\section{INTRODUCTION}

One of the main trends in the metalworking machine tool industry, which contributes to improving the processing efficiency, is to increase the cutting modes: the cutting speed (related to a spindle speed) and the thickness of the layer of material being removed in one pass of the tool (related to feed). As the possibility to increase the maximum processing modes to a large extent depends on the quality of fixture of a workpiece, it is necessary to provide the required value and stability of the clamping force over a wide range of spindle speeds. The process of clamping workpieces in a metalworking machine tool is carried out by using the clamping mechanism (CM). Since the $\mathrm{CM}$ is mounted on the spindle of the machine, it affects not only the efficiency of the workpiece clamping process but also the performance characteristics of the spindle assembly (spindle unit), in general. Any CM contains two main subsystems: "clamping actuator" (CA) and "clamping chuck". CA can be defined as an intermediate device for converting and transmitting energy in the form of a power flux from an energy source (ES) to the clamping chuck with functional dependence on it. Characteristics of the CA have a large influence on the main power characteristics of a $\mathrm{CM}$. Thus, the magnitude of the clamping force, stability of the clamping force under the influence of various perturbations such as the influence of centrifugal forces, deviation size of a clamping surface, heterogeneity of physical-mechanical characteristics of the material of the workpiece, and change of power supply characteristics are essential. Characteristics such as response time during mechanism operation, reliability of maintaining a tense system state while holding a workpiece, possibility of effective management and regulation of work of a CM are also important. A typical CA uses different methods to maintain the workpiece clamping force (tense system state) during processing. The need to use a large number of energy converters to create clamping forces by using of typical CA designs leads to additional energy losses, reduced reliability and efficiency of control of these mechanisms, increased sensitivity to external perturbations, complications of design, manufacturing and operation, etc. Not only does the efficiency of the energy converters work very often decrease with the increase of a spindle rotational speed, but their design also deteriorates the dynamic characteristic of a spindle assembly. The main researches for improving the productivity of the spindle unit in machine tools are dedicated to the detection of influence of bearings, optimal interaction of nodes, temperature influence, increasing vibration resistance and functional rigidity. Increasing the efficiency of creation clamping efforts can help to improve the processing efficiency through better utilization of the potential abilities of machine tools of the existing designs in particular. This explains the expediency of creating new designs of CA based on new structures that meet the above requirements.

\section{THE MAIN DISADVANTAGES OF TRADITIONAL DESIGNS OF ACTUATORS OF THE CLAMPING MECHANISM}

As a result of the analysis, the common problems of the main types of CA were identified and formulated. In the case when mechanical energy is supplied to the $\mathrm{CA}$ as an external axial force, there is an additional axial load on the spindle and its bearings. This can also lead to uncontrolled movement of the elements of the spindle assembly due to elimination gaps or elastic deformations. This reduces the accuracy of processing. Centrifugal inertia forces appear on the moving elements of CA converters (lever, wedge, etc) and constantly try to move them. It can cause uncontrolled negative changes in power characteristics of this mechanism during high-speed rotation of the spindle. The value of clamping effort created by a CA with the mechanisms of geometric closure depends on the variations in the size of the workpiece from its nominal values. The problem of a CA actuated by hydraulic pressure is that there is an occurrence of losses working fluid 
when bringing it to the rotating spindle. Also, the inertia forces acting on the liquid at high speeds counteract its displacement, which reduces the efficiency of the mechanism. Both types of CA require using large external mechanisms for preparing and supplying input energy (mechanical or hydraulical).

On the basis of the obtained information, ways of improving structure for the development of new CA designs are revealed:

- Characteristics of the energy converters should not depend on the influence of the centrifugal forces of inertia.

- Keeping the system's tense state while holding the workpiece must be ensured without supplying energy from the outside.

- The number of different types of energy converters should be minimal to simplify construction and reduce energy loss.

- Input energy must be supplied without external force interaction that can cause appearance of uncontrolled radial displacement of spindle units or offset of the rotation axis.

- Units that may complicate balancing of the spindle assembly or disturb it over a short period should be avoided.

- Change and adjustment of the characteristics of the clamping process must be available without disassembling the mechanism.

The safest and most economical CAs are those whose contour of force interaction is closed by braking inside the mechanism. CA of this kind can be disconnected from the power source after reaching the required clamping force and provide support even in the case of a power failure of a machine tool. Several basic types of self-braking gears can be used in the CA: screw, wedge, helix and cam. Self-braking mechanisms in the form of screw gearing have become most widespread as they are more economical in manufacturing and operation due to their simpler construction.

\section{DEVELOPMENT OF A TYPICAL STRUCTURE OF A NEW CLAMPING MECHANISM}

Following the identified requirements, a new CA structure was developed, with predictably better characteristics and where the input energy is electric. The widespread electrical actuator is an electromotor with rotation motion. The output force of a CA must be linear for work as a clamping chuck. To transform rotation motion in linear, it's reasonable to use a screw gear with the ability to self-brake (self-lock caused due to friction forces). It can keep a tensioned state of the system making it possible to hold a workpiece during processing without having to supply energy from an outer source. In such a way, the number of energy converters is reduced to two: one electromagnetic and one mechanical. Working characteristics of both converters do not depend on of centrifugal inertial forces and deviations of clamp surface size from nominal values. One variant of this structure is embodied in the patented CA design [Patent UA95323]. A feature of this design is the specific usage of an electromotor when a stator is attached to the body of the machine tool and the rotor is installed on a spindle. To create an effective CA based on this structure, it is necessary to identify the typical mathematical dependencies for this structure between the mass-geometric parameters of the elements of the mechanism, and the kinematic and power characteristics. That is why, it is necessary to establish the basic dependencies that describe the characteristics of displacement of the CA output link (value of displacement, velocity) and the amount of energy (value of output effort) spent to clamp the workpiece in the $\mathrm{CM}$ with $\mathrm{CA}$ of the proposed design.
The block diagram of a typical collet $\mathrm{CM}$ in which the proposed CA is used can be represented by the following set of structural elements (Fig. 1): the energy source ES is usually an industrial electrical network with voltage $U$; the switching device $(\mathrm{SD})$, which controls the operation of the drive motor $\mathrm{D}$, consisting of a stator and rotor (on the shaft of which rotation moment $\mathrm{Ma}_{0}$ occurs ); the drive self-braking mechanism (DSBM) changes the movement characteristics of the output link of the drive motor $M$ and captures the tense state of the CM after it is switched off; a clamping chuck (CC), which directly fixes the object of the clamping (OC) with $\mathrm{Fr}_{2}$ radial force.

Several types of control of engine $D$ can be implemented in this structure of CM to automatically adjust the parameters of the $\mathrm{CM}$, for which different backward linkages I, II and III (Fig. 1) are commonly used. The control system of electrical CA, the core element of which is the SD switching device, must ensure that in addition to $\mathrm{Fr}_{2}$ clamping force control, there is also mutual locking with the main drive of the machine, feed and auxiliary movements.

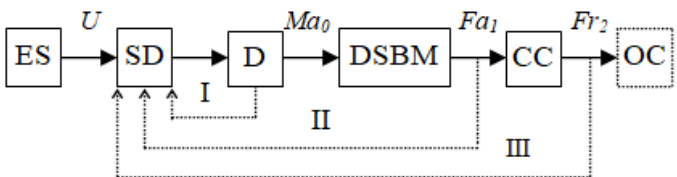

Fig. 1. Block diagram of a collet $\mathrm{CM}$ with the electrical $\mathrm{CA}$ and self- braking mechanism

The effective control of the operation of the CM with the electrical CA can be performed using a switching device (SD) by changing the stator current parameters that determine the rotor motion characteristics. The control signal may include characteristic operational values (displacement and force interaction) of the various CM subsystems and is transmitted to the SD through separate backward linkages I, II and III (Fig. 1). This improves the reliability and efficiency of the clamping process control. The influence of centrifugal inertia forces of unbalanced moving $\mathrm{CM}$ elements on changing the value of clamping force $\mathrm{Fr}_{2}$ can be determined from a function where the actual spindle speed is used as a variable.

Obtaining a predetermined value of clamping force $\mathrm{Fr}_{2}$ in $\mathrm{CM}$ with electrical CA can be carried out based on several principles of its control system organization. The simplest is the principle of control without deliberately limiting engine running time. In this case, the electrical CA drive motor operates in stopper mode operation until the rotor is stopped under the influence of resistance forces (overturning mode), which corresponds to the maximum value of the tense system state for the given power supply characteristics. This is reflected in the characteristics of the motor current of the motor $D$ and gives a signal for the switching device to switch it off. The system remains in the tense state which is supported by the self-braking mechanism (DSBM). Overloading of the drive motor during stopping is acceptable since it has a small duration. Other clamping process control principles use signals from sensors giving information about the value of displacement and force interaction in the CM subsystems to limit the clamping force and provide controlled stopping of the electrical $\mathrm{CA}$ engine. In these cases, the response time of the control equipment (relay and sensors) must be taken into account. The possibility of using different control schemes of electrical $C A$ increases the efficiency of its usage in the CM for different technological equipment and different operation conditions.

In the proposed design of an electric CA (Fig. 2), the rotor of 
the induction motor $D$ is located on the threaded surface of the spindle with the possibility of their mutual movement, and the stator of electric CA is rigidly connected to the housing of the spindle assembly. Energy supply to the rotating spindle is carried out without contact - due to the influence of the stator electromagnetic torque. At the initial moment of operation of this $\mathrm{CM}$, the rotor of the motor $\mathrm{D}$ electrical $\mathrm{CA}$ has an angular velocity relative to the stator, which is equal to the speed of rotation of the spindle. Rotation acceleration or deceleration of the rotor of motor $D$ and its movement in relation to the spindle occurs under the action of the electromagnetic moment of stator due to the activation of motor $\mathrm{D}$. This also results in the axial movement of the rotor on the spindle threaded surface with a force $\mathrm{Fa}_{1}$, which is the output force of electric $\mathrm{CA}$.

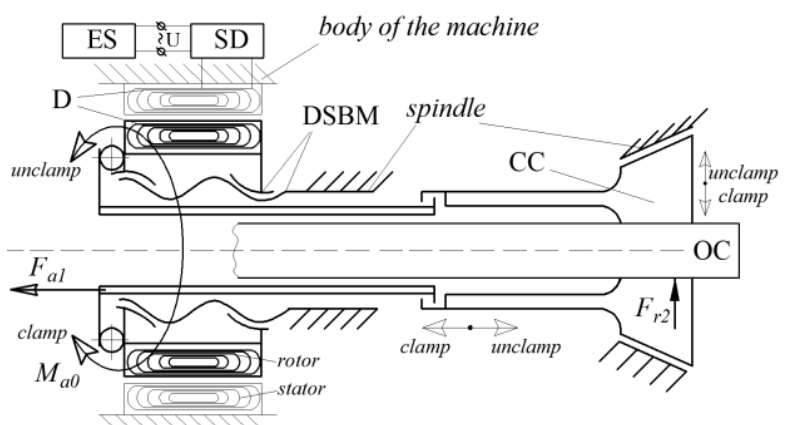

Fig. 2. Schematic diagram of electric $C A$ according to the patent of Ukraine № 95323

\section{IDENTIFICATION OF ANALYTICAL DEPENDENCIES}

The mathematical description of a multi-mass dynamic model, which reflects the working process of the $\mathrm{CM}$ with the proposed electric CA, can be carried out in accordance with the calculation scheme (Fig. 3a). Detailed calculations of this type are related to the difficulties in computation and their volume. Therefore, for practical calculations and further analytical studies, the multi-mass system is reduced to a single-mass system (Fig. $3 b, c)$ with the equivalent moment of inertia $J_{\Pi}$ and the equivalent torque $M_{n}=M_{0}$. On the scheme (Fig. $3 b, c$ ), the influence of forces of potential character (forces of elasticity) is reflected by the action of elastic elements with the corresponding stiffness coefficient $C_{n \pi}$, $C_{n p}, C_{\kappa}$.

\subsection{Development of calculation schemes}

It is reasonable to consider $\mathrm{CM}$ as a system in two cases: during the clearance $\Delta$ adjustment, when mainly active forces of dissipative character are acting in the system, and during the creation of tension of the elastic system of CM (Fig. 3 b) when the forces of potential character dominate. The analysis of the clamping process in the $\mathrm{CM}$ with the electric $\mathrm{CA}$ (Fig. $3 \mathrm{~b}, \mathrm{c}$ ) should also take into account the forces of elasticity of the collet petals with the rigidity $C_{n л}$, stiffness of electric $C A-C_{n p}$, and contact stiffness in the joints of the collet chuck cartridge $C_{\kappa}$. The study of the CA in the SM (Fig. 3) requires consideration of the kinematic characteristics of two interacting mechanical energy converters: a screw self-braking mechanism (DSBM) and a collet (CC).

In a screw-driven self-braking mechanism, the rotation rotor of $D$ (which works like a nut in a screw mechanism) with an angular velocity $\omega_{\partial}$ ensures its translational motion with the speed $V_{m}$ transmitted to the output link of CA - "clamp pipe". The relation- ship between these kinematic parameters can be established based on the equality between the rotation angle of the motor rotor $\varphi_{\partial}$ and the axial movement of the "clamp pipe" $x_{m}$ in one complete turn of the rotor $\phi_{\partial} / x_{m}=2 \cdot \pi / h$ or

$x_{m}=\frac{\phi_{\partial} \cdot h}{2 \pi}$,

where $h$ is the pitch of the thread.

a)

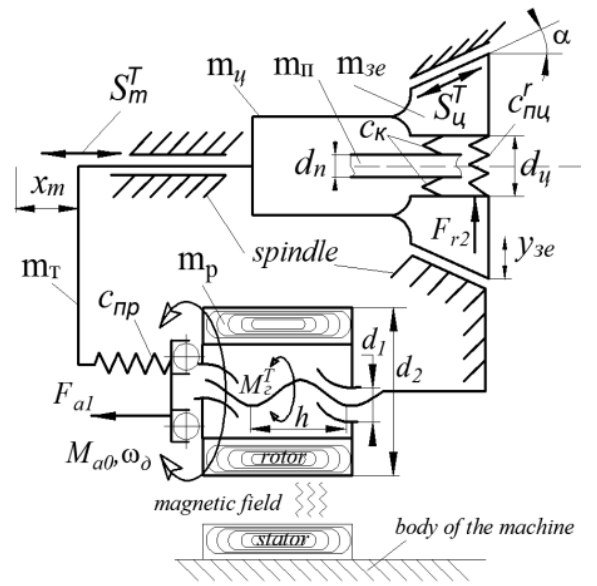

b)

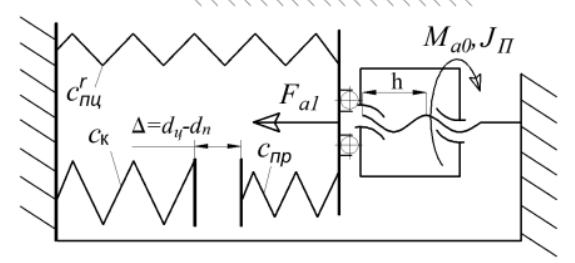

c)

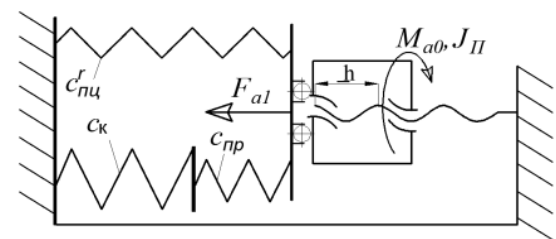

Fig. 3. Scheme of the CM: a) calculation scheme; b) simplified dynamic models during elimination gaps; c) simplified dynamic models when creating tension in the system

After differentiating the left and right parts of equations (1) in time, we obtain $\partial x_{m} / \partial t=\left(\partial \phi_{\partial} / \partial t\right) \cdot h / 2 \pi$,

$V_{m}=\omega_{\partial} \cdot \frac{h}{2 \pi}=0.5 \omega_{\partial} \frac{h}{\pi}=\omega_{\partial} \cdot 0.5 d_{1} \cdot \operatorname{tg} \psi$,

where $d_{1}$ is the diameter of the thread, $\psi$ is the lead angle of the screw and $\omega_{\partial}$ is the angular velocity of the rotor of the engine.

Express the value of $i_{s}=0.5 d_{1} \cdot \operatorname{tg} \psi$ and substitute it in the equation (2). In that way, we get $V_{m}=\omega_{\partial} \cdot i_{s}$ or:

$V_{m}=\dot{\phi}_{\partial} \cdot i_{2}$.

The movement of the collet chuck in the axial direction with velocity $V_{\mathrm{T}}$ provides radial movement of the clamping elements of the collet with velocity $V_{3 E}$. The relationship between these kinematic parameters can be established based on the equality of relation of axial displacement $x_{m}$ of the "clamp pipe" together with the body of the collet to the radial displacement $y_{3 e}$ of the collet surfaces intended to contact the object of fixation OC, $x_{m} / y_{3 e}=$ $\operatorname{ctg} \alpha$ from this it derives that:

$y_{3 e}=\frac{x_{m}}{\operatorname{ctg} \alpha}$,

$\partial x_{m} / \partial t=\left(\partial t_{3 e} / \partial t\right) \cdot \operatorname{ctg} \alpha, V_{m}=V_{3 e} \cdot \operatorname{ctg} \alpha$ where $\alpha$ is half of cone angle of collet. $i_{y}=\operatorname{ctg} \alpha$, therefore, 
$V_{3 e}=\frac{V_{m}}{i_{u}}$.

After adding (3) to (5), we obtain

$V_{s e}=\frac{\dot{\phi}_{\partial} \cdot i_{z}}{i_{u}}=\dot{\phi}_{\partial} \frac{i_{2}}{i_{u}}$

Analysis of the energy balance of the CM with proposed electrical CA (Fig. 3, a) during the workpiece clamping can be done by comparing the energy $E_{3}$ consumed by the $\mathrm{CM}$ during a clamping process in general to the effective clamping energy $E_{e \Phi}$ consumed in the contact zone of collet surfaces with the object of fixation $\mathrm{OC}$ to create contact deformations. It should be noted that, unlike with pneumatic and hydraulic actuators, there is no component of energy lost for maintaining the system's tense state. Therefore, the calculation of the total energy required for operation of the $\mathrm{CM}$ can be carried out by the formula:

$E_{3}=E_{n u}+A_{m p}+E_{e q}$,

where $E_{\eta M}$ is the energy consumed for the movement of the masses of a unit CM, $A_{m p}$ is the work (energy equivalent) performed to overcome friction forces, and $E_{e \Phi}$ is the effective clamping energy consumed in the contact zone of collet surfaces with the object of fixation $\mathrm{OC}$ to create contact deformations.

\subsection{Detection of dependencies between geometrical-mass parameters and power and kinematic characteristics of the mechanism}

The efficiency coefficient of the mechanical part of the studied CM (Fig. 2) can be determined by the formula:

$\eta_{3 M}=\frac{E_{e \phi}}{E_{n u}+A_{m p}+E_{e \phi}}$.

In this case, $E_{n M}$ is the sum of the kinetic energy of mechanism links, which are rotating or have reciprocating motion:

$$
\begin{aligned}
& E_{n u}=\sum_{\mathrm{i}=1}^{\mathrm{i}=\mathrm{n}} \frac{m_{i} V_{i}^{2}}{2}+\sum_{i=1}^{i=n} \frac{I_{i} \omega_{i}^{2}}{2}, \\
& E_{n u}=\frac{m_{o c}}{2} V_{o c}^{2}+\frac{m_{p D}}{2} V_{p d}^{2}+J_{\partial} \frac{\omega_{\partial}^{2}}{2},
\end{aligned}
$$

$m_{o c}=m_{p}+m_{m}+m_{y}$, where $V_{o c}, m_{o c}$ are, respectively, linear velocity (equal to "clamp pipe" velocity $V_{m}$ ) and total mass of CM elements moving in the axial direction: motor rotor, "clamp pipe", collet of the clamping mechanism; $m_{p \partial}, V_{p \partial}$ are, respectively, the mass and linear velocity of the $\mathrm{CM}$ elements moving in the radial direction, in this case $m_{p d}=m_{3 e}$, where $m_{3 e}$ is the mass of clamping elements of the collet and $V_{p \partial}=V_{3 e}$, where $V_{3 e}$ is the radial speed of movement of clamping elements of the collet; $J_{\partial}, \omega_{\partial}$ are the moment of inertia of the rotor and its angular velocity.

Analytical dependencies (6) do not take into account the mass of the workpiece that moves with the collet, since it can change significantly in each specific cycle of fixation, which distorts the value of the efficiency as an inherent characteristic of the $\mathrm{CM}$ of the investigated structure.

Moment of inertia of the engine rotor $J_{\partial}=m_{p}\left(d_{1}^{2}+d_{2}^{2}\right) / 8$. According to (2), (5) between $\omega_{\partial}, V_{3 e}$ and $V_{m}$, there are certain kinematic dependencies that are determined according to the calculation scheme: $V_{o c}=V_{m}=\omega_{\partial} \cdot 0.5 d_{1} \cdot \operatorname{tg} \psi, V_{m}=V_{3 e}$. ctg $\alpha, V_{p \sigma}=V_{3 e}=\omega_{\partial} \cdot 0.5 d_{1} \cdot \operatorname{tg} \psi \cdot \operatorname{tg} \alpha$. Then (9) can be written in the following form:

$$
E_{n u}=\frac{1}{8}\left(\omega_{\partial} d_{1} \operatorname{tg} \psi\right)^{2}\left(m_{o c}+m_{p o} \operatorname{tg}^{2} \alpha+0.5 \frac{m_{p}\left(d_{1}^{2}+d_{2}^{2}\right)}{\left(d_{1} \operatorname{tg} \psi\right)^{2}}\right)
$$

The work $A_{m p}$ (energy equivalent) performed to overcome the friction forces in $\mathrm{CM}$ consists of the following works: friction forces $S_{m}^{T}$ of the "clamp pipe" in contact with the spindle during their mutual movement on the value of the "clamp pipe" stroke length $x_{m}$; the friction forces $S_{\mu}^{T}$ in contact with the collet cone with the spindle during their mutual movement on the value of the collet stroke length $x_{y}=x_{m} / \cos \alpha$; given the proximity of the value $\cos \alpha$ from 0.97 to 1 , the calculations may be simplified to approximately $x_{y} \approx x_{m}$; friction in the screw gear of the DSBM, which create the torque of friction forces $M_{S}^{T}$ when turning with the angle $\phi_{\partial}$.

$$
A_{m p}=S_{m}^{T} \cdot x_{m}+S_{u}^{T} \cdot x_{m}+M_{2}^{T} \cdot \phi_{\partial} .
$$

The moment created by the forces of friction in the screw gear $M_{S}^{T}$ can be defined as the action of the tangential component of the friction forces $F_{t}$ with the shoulder equal to half the average diameter of the screw thread $M_{s}^{T}=0.5 d_{1} F_{t}$. The torque generated by the friction in the connection bearings of the rotor to the "clamp pipe" is extremely small compared to $M_{s}^{T}$, so we can neglect its magnitude. The tangential component $F_{t}$ of the friction force depends on the axial load $F a_{1}$ arising at the output of the gear. $F_{t}=F a_{1} \cdot \operatorname{tg} \phi^{T}$, where $\phi^{T}$ is the angle of friction in the threaded connection. Thus, the moment created by the friction forces in the screw transmission under load $F a_{1}$ can be determined from the following dependency:

$M_{2}^{T}=0.5 \cdot d_{1} \cdot F a_{1} \cdot \operatorname{tg} \phi^{T}$.

The friction force between the surfaces of the collet cone and the spindle cone with the angle $2 \alpha$ is determined by the formula:

$S_{u}^{T}=\frac{z \times F a_{1}}{\cos \alpha+(\sin \alpha) / f_{u}}$,

where $z$ is the number of the collet segments and $f_{\mu}$ is the coefficient of friction in the conical coupling of the collet with the spindle.

The force $S_{m}^{T}$ of friction "clamp pipe" with a spindle:

$S_{m}^{T}=g \cdot m_{2} \cdot f_{m}$,

where $g$ is the free fall acceleration, $m_{2}=m_{m}+m_{n}$ is the mass equal to the sum of the mass of the "clamp pipe" and the workpiece (as a rod), and $f_{m}$ is the coefficient of friction in the coupling of the "clamp pipe" with the spindle.

Using (12), (13) and (14), we write (11) as follows:

$$
A_{m p}=F a_{1} \frac{\phi_{o} h}{2 \pi}\left(\frac{g m_{2} f_{m}}{F a_{1}}+\frac{z}{\cos \alpha+(\sin \alpha) / f_{u}}+\operatorname{tg} \phi^{T} \cdot \operatorname{ctg} \psi\right) \text {. }
$$

Effective clamping energy $E_{e \Phi}=z \int_{0}^{h_{k}} F r_{2}\left(h_{k}\right) d h_{k}$, where $h_{k}$ is the convergence in contact points between collet and spindle under the action of a normal clamping force, and the formula for determining it was obtained by experimentally $h_{k}=$ $f\left(S^{k}, F r_{2}, \Delta d\right)$, where $S^{k}$ is the area of contact of the respective ribbed surfaces of the collet with the object of fixation. According to literary sources:

$$
E_{e \phi}=\frac{m \cdot S^{\kappa} \cdot z \cdot h_{k}}{A_{M}^{(1 / m)} \cdot(1+m)},
$$

where $A_{M}, m$ are coefficients selected according to the conditions 
of the clamping process. For clamping conditions with a full fit of the clamping elements and object of fixation, $m=1, A_{M}=0.13$ and dependency (16) takes shape:

$$
E_{e \phi} \approx 3.82 \cdot S^{\kappa} \cdot z \cdot h_{k} \text {. }
$$

Compute of the value of the work efficiency coefficient of clamping mechanisms with the electromechanical actuator derives from putting dependencies (10), (15), (17) into (8). Choosing appropriate characteristics of the elements of the new structure conduces to elimination of the problems related to increasing efficiency of fixation of a workpiece for high-performance machining. The obtained analytical dependencies (10), (15), (17), (18) make it possible to identify values that describe the efficiency of the $\mathrm{CM}$ and depend on some geometric and mass parameters of the elements of the mechanism:

$$
\begin{aligned}
& \eta_{3 M}=3.82 S^{\kappa} z h_{k} / \times\left(m_{o c}+m_{p o} t^{2} \alpha+0.5 \frac{\frac{1}{8}\left(\omega_{\partial} d_{1} \operatorname{tg} \psi\right)^{2} \times}{\left.\left(d_{1}+t g\right)^{2}\right)}\right) \\
& +F a_{1} \frac{\phi_{\partial} h}{2 \pi}\left(\frac{g m_{2} f_{m}}{F a_{1}}+\frac{z}{\cos \alpha+(\sin \alpha) / f_{u}}+\operatorname{tg} \phi^{T} \cdot \operatorname{ctg} \psi\right) \\
& +3.82 \cdot S^{\kappa} \cdot z \cdot h_{k} .
\end{aligned}
$$

To illustrate the example of using the dependence (18), the efficiency of the prototype mechanism [16] is calculated. The separated components (10), (15), (17) of the expression (18) are calculated. In accordance with the dependence (10), the value of $E_{n M}$ is determined at the parameters of the mechanism: $\omega_{\partial}=$ $314 \mathrm{rad} / \mathrm{s}, d_{1}=0.09 \mathrm{~m}, \operatorname{tg} \psi=0.01, m_{o c}=3 \mathrm{~kg}, m_{p \partial}=$ $0.4 \mathrm{~kg}, \operatorname{tg} \alpha=0.268, m_{p}=2.4 \mathrm{~kg}, d_{2}=0.14 \mathrm{~m}, E_{n M}=$ $\frac{1}{8}(314 \cdot 0.09 \cdot 0.01)^{2} \times$

$$
\left(3+0.4 \cdot 0.268^{2}+0.5 \frac{2.4\left(0.09^{2}+0.14^{2}\right)}{(0.09 \cdot 0.01)^{2}}\right)=409.7 \mathrm{Nm} \text {. }
$$

In accordance with the dependence (15), the value of $A_{m p}$ is determined at the parameters of the mechanism: $F a_{1}=$ $1200 \mathrm{~N}, \quad \phi_{\partial}=44 \mathrm{rad}, h=2 \cdot 10^{-3} \mathrm{~m}, g=9.81 \mathrm{~m} / \mathrm{s}^{2}$, $m_{2}=3.2 \mathrm{~kg}, \quad f_{m}=0.3, \quad z=3, \quad \alpha=15^{\circ}, \quad \operatorname{tg} \phi=0.2$, $\operatorname{ctg} \psi=5, \quad f_{\mu}=0.1, \quad A_{m p}=1200 \frac{44 \cdot 2 \cdot 10^{-3}}{2 \pi} \times\left(\frac{9.81 \cdot 3.2 \cdot 0.3}{1200}+\right.$ $\left.\frac{3}{0.966+0.259 / 0.1}+0.2 \cdot 5\right)=31.1 \mathrm{Nm}$.

In accordance with the dependence (17), the value of $E_{e \Phi}$ is determined at the parameters: $S^{k}=95 \cdot 10^{-5} \mathrm{~m}^{2}, z=3$, $h_{k}=100 \mathrm{kN} / \mathrm{m}, E_{e \Phi}=3.82 \cdot 10^{-5} \cdot 3 \cdot 100 \mathrm{kNm}$.

In accordance with the dependence (8), the efficiency coefficient $\eta_{3 \mathrm{M}}$ of the CM [16] is:

$$
\eta_{3 M}=\frac{1080}{409.7+31.1+1080}=0.71 \text {. }
$$

The obtained dependences (18) allow to carry out the analysis of influence of the CM parameters on its work efficiency. It enhances the efficiency of design work allowing to find more appropriate parameters easier. Thus, (18) demonstrates that one of the promising ways to increase the efficiency of the $\mathrm{CM}$ is to reduce the value of moving masses $\mathrm{m}_{\mathrm{oc}}, \mathrm{m}_{p}, \mathrm{~m}_{2}$ and other. Reduction of the mass $m_{p}$ of the motor rotor can be achieved through the use of modern high-torque motors with a rotor that has a much smaller mass, outer diameter $d_{2}$ and moment of inertia. The weight of the elements of the $\mathrm{CM}$ can be reduced through the use of composite and polymeric materials which weigh three up to four times less. For example, the rod connecting the clamp drive and the clamping chuck which is made in the form of a pipe can be made of carbon fibre. Using the formula (18) gives enhanced opportunities for the designer to make informed decisions about the feasibility of using new structural elements and materials, improving the quality of surfaces machining to reduce the coefficients of friction $f_{m}, f_{\mu}$. That is, (18) makes it possible to evaluate the technical solutions in terms of comparing the costs of their implementation and the magnitude of productivity gains. Determining the appropriate periods between maintenance is dependent on the allowable amount of loss of the mechanism efficiency due to its normal wear. The level of degradation of the friction surfaces and the change of the friction coefficient allows to calculate the level of reduction of the mechanism productivity by means of (18) and to make more accurate decisions about the need for maintenance or overhaul.

The proposition would change the parameters $\mathrm{m}_{\mathrm{oc}}$ and $\mathrm{m}_{\mathrm{oc}}$ by making the parts of the mechanism from titanium with the purpose of improving. The magnitude of the change in the part's mass when changing its material while keeping its strength can be determined by comparing the coefficients of specific strength of each material. The specific strength is a material's strength divided by its density. In the manufacture of parts of titanium, their weight will be twice less than that made of alloy steel and two and a half times less compared to high-quality carbon steel with providing the same strength. The masses $\mathrm{m}^{\prime}{ }_{\text {oc }}=1.5 \mathrm{~kg}$ and $\mathrm{m}^{\prime}{ }_{\mathrm{p}}=1.2 \mathrm{~kg}$ of the parts which are made of new material are reduced by $50 \%$ comparatively with the previous variant. The diameter of the rotor $\mathrm{d}_{2}^{\prime}=0.13 \mathrm{~m}$ can be reduced by $7 \%$ by using more expensive special manufacturing technologies. The parameters $m_{o c}, m_{p}$ and $d_{2}$ have an influence on the value $E_{n M}$, and consequently on the efficiency $\eta_{3 M}$. The value of $E_{n M}^{\prime}$ determined at the new parameters of the mechanism is:

$$
\begin{aligned}
& E_{n M}^{\prime}=\frac{1}{8}(314 \cdot 0.09 \cdot 0.01)^{2} \times \\
& \left(1,5+0.4 \cdot 0.268^{2}+0.5 \frac{1.2\left(0.09^{2}+0.13^{2}\right)}{(0.09 \cdot 0.01)^{2}}\right)=184.88 \mathrm{Nm} .
\end{aligned}
$$

The value of the mechanism's efficiency with new parameters $\eta_{3 \mathrm{M}}^{\prime}$ can be calculated with (18): $\eta_{3 \mathrm{M}}^{\prime}=\frac{1080}{184.88+31.1+1080}=0.833$

The proposed changes of the masses and geometrical parameters resulted in the improvement of the efficiency coefficient $\eta_{3 \mathrm{M}}^{\prime}=0.833$ comparatively with the previous $\eta_{3 \mathrm{M}}=0.71$ by $17 \%$.

\section{CONCLUSION}

As a result of the evaluation of traditional $\mathrm{CM}$ design function, the main disadvantages, and factors limiting clamping process efficiency and in particular at high speeds of rotation, have been identified. Based on the obtained information, the characteristics of the elements and the structure of the CM with improved characteristics are revealed and described. Following the example of the patented mechanism design of this type, function is considered, and the calculation scheme and simplified dynamic models describing different stages of its work are made. It is revealed that for the mechanisms of this type, the first stage of operation which is related to elimination of the gaps is distinguished and displayed in the relatively enlarged rotation angle of the input link (rotor) in the free-running mode. Therefore, the special feature of the mechanism of the proposed structure is in the possibility of using the work of inertia forces of the input link, the magnitude of which increases over its acceleration period, can be adjusted and de- 
pends on the magnitude of the rotation angle of the rotor during free-running. Also, by changing the geometrical-mass characteristics (moment of inertia) of the rotating input link, the force characteristics of the clamping process can be corrected.

The calculation scheme was formulated to determine massgeometric parameters of mechanism elements should influence the main characteristics of the clamping mechanisms of this type. Based on the revealed information, dependencies for determining the specific kinematic and dynamic characteristics and the value of the work efficiency coefficient of the clamping mechanism of this type were found. The obtained dependencies are among the necessary prerequisites for creating an automatic system for calculating the work characteristics of $\mathrm{CM}$ of this type. Usage of obtained analytical dependence enhances the efficiency of design work and operation of the CM due to the possibility of selecting the mass and geometrical parameters of the elements.

\section{REFERENCES}

1. Alquraan T., Kuznetsov Yu., Tsvyd T. (2016) High-speed clamping Mechanism of the CNC lathe with compensation of centrifugal forces, Procedia engineering, 150, 689-695.

2. An J., Jiamin C., Wenguo Y. (2019), Measurement of spindle radial error based on target trajectory tracking, Measurement, 146,179-185.

3. Bediz B., Gozen B.A., Korkmaz E., Ozdoganlar O. B. (2014), Dynamics of ultra-high-speed (UHS) spindles used for micromachining, International Journal of Machine Tools and Manufacture, 87, 27-38.

4. Budniak Z. (2015), Modelling and numerical analysis of assembly system, Acta mechanica et Automatica, 9(3), 145-150.

5. Chao Xu, Jianfu Z., Pingfa F. (2014), Characteristics of stiffness and contact stress distribution of a spindle-holder taper joint under clamping and centrifugal forces, International Journal of Machine Tools \& Manufacture, 82-83, 21-28.

6. Dogariu C., Bardac D. (2014), Prediction of the structural dynamic behavior of high speed turning machine spindles, Applied Mechanics and Materials, 555, 567-574.

7. Estrems M., Arizmendi M., Cumbicus W.E., López A. (2015), Measurement of clamping forces in a 3 jaw chuck through an instrumented aluminium ring, Procedia Engineering, 132, 456-463.

8. Fedorynenko D., Sapon S., Boyko S. (2016), Accuracy of spindle units with hydrostatic bearings, Acta Mechanica et Automatica, 10(2), 117-124

9. Fedorynenko D., Sapon S., Boyko S., Urlina A. (2017), Increasing of energy efficiency of spindles with fluid bearings, Acta Mechanica et Automatica, 11(2), 204-209.

10. Foremny E., Schenck C., Kuhfuß B. (2016), Dynamic Behavior of an Ultra Precision Spindle used in Machining of Optical Components, Procedia CIRP, 46, 452-455.

11. Grama S.N., Mathur A., Badhe A.N. (2018), A model-based cooling strategy for motorized spindle to reduce thermal errors, International Journal of Machine Tools and Manufacture, 132, 3-16.

12. Grossi N., Scippa A., Montevecchi F. (2016), A novel experimental-numerical approach to modeling machine tool dynamics for chatter stability prediction, Journal of advanced mechanical design systems and manufacturing, 10(2), \#15-00547.

13. Harris P., Linke B., Spence S. (2015), An Energy Analysis of Electric and Pneumatic Ultra-high Speed Machine Tool Spindles, Procedia CIRP, 29, 239-244.

14. Jia Q., Li B., Wei Y., Chen Y., Yuan X. (2016), Axiomatic Design Method for the Hydrostatic Spindle with Multisource Coupled Information, Procedia CIRP, 53, 252-260.

15. Kono D., Mizuno S., Muraki T., Nakaminami M. (2019), A machine tool motorized spindle with hybrid structure of steel and carbon fiber composite, CIRP Annals, 68(1), 389-392.
16. Li, W.; Zhou, Z. X.; Xiao, H. (2015), Design and evaluation of a high-speed and precision microspindle, International journal of advanced manufacturing technology, 78(5), 997-1004.

17. Liu T., Gao W., Zhang D., Tian Y. (2017), Analytical modeling for thermal errors of motorized spindle unit, International Journal of Machine Tools and Manufacture, 112, 53-70.

18. Longfei Z., Jun Z., Chao Z. (2019), A new method for field dynamic balancing of rigid motorized spindles based on real-time position data of CNC machine tools, International journal of advanced manufacturing technology, 102 (5-8), special edition, 1181-1191.

19. Matsubara A., Tsujimoto S., Kono D. (2015), Evaluation of dynamic stiffness of machine tool spindle by non-contact excitation tests, CIRP Annals, 1.V. 64(1), 365-368.

20. Mori K., Bergmann B., Kono D., Denkena B., Matsubara A. (2019), Energy efficiency improvement of machine tool spindle cooling system with on-off control, CIRP Journal of Manufacturing Science and Technology, 25, 14-21.

21. Postel M., Aslan D., Wegener K., Altintas Y. (2019), Monitoring of vibrations and cutting forces with spindle mounted vibration sensors, CIRP Annals, 68(1), 413-416.

22. Prydalnyi B. (2020), Characteristics of electromechanical clamping mechanism with asynchronous electric motor, International Conference Mechatronic Systems and Materials (MSM), 1-5.

23. Rabréau C., Ritou M., Le Loch S., Furet B. (2017), Investigation of the Evolution of Modal Behavior of HSM Spindle at High Speed, Procedia CIRP, 58, 405-410.

24. Ritou M., Rabréau C., Le Loch S., Furet B., Dumur D. (2018), Influence of spindle condition on the dynamic behavior, CIRP Annals, 67(1), 419-422

25. Shaoke W., Jun H., Fei D. (2019), Modelling and characteristic investigation of spindle-holder assembly under clamping and centrifugal forces, Journal of mechanical science and technology, 33(5), 2397-2405

26. Thorenz B., Westermann H.-H., Kafara M., Nützel M., Steinhilper R. (2018), Evaluation of the influence of different clamping chuck types on energy consumption, tool wear and surface qualities in milling operations, Procedia Manufacturing, 21, 575-582.

27. Wang H.J. (2013), Study of dynamics characteristics for precision motor spindle system, Advanced materials research, 819, 389-392.

28. Xu C., Zhang J., Feng P., Yu D., Wu Z. (2014), Characteristics of stiffness and contact stress distribution of a spindle-holder taper joint under clamping and centrifugal forces, International Journal of Machine Tools and Manufacture, 82-83, 21-28.

29. Yadav M.H., Mohite S.S. (2018), Controlling deformations of thinwalled Al 6061-T6 components by adaptive clamping, Procedia Manufacturing, 20, 509-516.

30. Yang Y., Zhang W.H., Ma Y.C., Wan M. (2015), Generalized method for the analysis of bending, torsional and axial receptances of toolholder-spindle assembly, International Journal of Machine Tools and Manufacture, 99, 48-67.

31. Yuan S.M. (2014), The analysis of static and dynamic characteristics of motorized high-speed spindle based on sensitivity analysis of fem model, Applied mechanics and materials, 43, 376-381.

32. Zabielski R., Trochimczuk R. (2011), Wybrane problemy projektowania wysokoobrotowych elektrowrzecion frezarskich o niestandardowym łożyskowaniu, Acta Mechanica et Automatica, 5(1), 131-136.

33. Zhang S., Yu J., To S., Xiong Z. (2018), A theoretical and experimental study of spindle imbalance induced forced vibration and its effect on surface generation in diamond turning, International Journal of Machine Tools and Manufacture, 133, 61-71.

Acknowledgement: The work has been accomplished under the research activity of Borys Prydalnyi at the Faculty of Mechanical Engineering, Bialystok University of Technology, Poland, in frame of the PROM Project: "International scholarship exchange of $\mathrm{PhD}$ candidates and academic staff" within the Operational Programme Knowledge Education Development, co-financed from the European Social Fund.

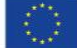

